\title{
Short Polarization Filter in Pillar-Based Photonic Crystals
}

\author{
Abigaël A. M. Kok, Student Member, IEEE, Erik Jan Geluk, Fouad Karouta, Senior Member, IEEE, \\ Jos J. G. M. van der Tol, Roel Baets, Fellow, IEEE, and Meint K. Smit, Fellow, IEEE
}

\begin{abstract}
The strong polarization dependence of twodimensional photonic crystals is exploited for polarization filtering. The device with a length of $3.9 \mu \mathrm{m}$ is integrated in a photonic integrated circuit based on InP waveguide technology. The average transmissions in the wavelength range from 1530 to $1570 \mathrm{~nm}$ are $-8.7 \mathrm{~dB}$ for transverse-magnetic polarization and $-26.5 \mathrm{~dB}$ for transverse-electric polarization.
\end{abstract}

Index Terms-Optical waveguide components, photonic crystals, polarization.

\section{INTRODUCTION}

$\mathbf{T}$ WO-DIMENSIONAL (2-D) photonic crystals (PhCs) have been subject to extensive research in the past years, offering a huge potential for integration in photonic integrated circuits. They can be used to miniaturize existing integrated optical devices, e.g., bends, microcavities, add-drop filters, and band edge lasers. Furthermore, their special properties can be used to design devices based on new principles. An example of such a device is a polarization filter. The polarization sensitivity of $\mathrm{PhCs}$ makes it possible to significantly reduce the foot print of polarization filters.

Photonic integrated circuits can be realized in different material systems, depending on the applications. However, indium phosphide (InP) technology is the only platform to monolithically integrate active and passive devices for use in the telecom wavelength window $(1530-1570 \mathrm{~nm})$. The classical waveguides of InP photonic integrated circuits are based on total internal reflection; light is confined to the indium gallium arsenide phosphide (InGaAsP $Q[1.25]$ ) core, which has a higher refractive index than the InP cladding layers $\left(n_{\mathrm{InGaAsP}}=3.3640\right.$ and $n_{\mathrm{InP}}=3.1693$ at a wavelength of $1550 \mathrm{~nm}$ [1]). In 2-D PhCs, the in-plane confinement is created by the photonic bandgap properties of the crystal, whereas the light is mainly confined out-of-plane by total internal reflection in the semiconductor layer stack.

In photonic integrated circuits for telecommunication networks, polarization control is of increasing importance [2]. This

Manuscript received March 7, 2008; revised April 5, 2008. This work was supported by NanoNed, a technology program of the Dutch Ministry of Economic Affairs via the foundation STW.

A. A. M. Kok, E. J. Geluk, F. Karouta, J. J. G. M. van der Tol, and M. K. Smit are with the COBRA Research Institute, Eindhoven University of Technology, 5600 MB Eindhoven, The Netherlands (e-mail: j.j.g.m.v.d.tol@tue.nl).

R. Baets is with the Department of Information Technology, Ghent University-IMEC, $9000 \mathrm{Gent}$, Belgium. He is also with the COBRA Research Institute, Eindhoven University of Technology, 5600 MB Eindhoven, The Netherlands.

Digital Object Identifier 10.1109/LPT.2008.926861 control is obtained by the integration of polarization splitters, converters, and filters in the photonic circuit. Polarization splitters based on classical waveguide technology are relatively long devices, in the order of millimeters [3], [4]. The highly polarization-dependent nature of $\mathrm{PhCs}$ enables the use of them in short polarization splitters, significantly reducing their size [5]-[7]. However, if only a filtering function is needed, the use of relatively long and complicated polarization splitters can be avoided. In this work, the strong polarization dependence of 2-D $\mathrm{PhCs}([8])$ is used to investigate a transverse-electric (TE) polarization filter based on a pillar $\mathrm{PhC}$ waveguide in a square lattice of high-index pillars. Transverse-magnetic (TM)-polarized light is defined as the polarization that has its electric field vector parallel to the pillars, and TE-polarization has its electric field vector in the plane of the chip. The device is integrated in a classical photonic integrated circuit on an InP substrate with a 500-nm-thick InGaAsP core layer and a 1- $\mu$ m-thick InP top cladding. The layer stack of the pillar $\mathrm{PhC}$ is compatible with that of the classical photonic integrated circuit, and so is the fabrication technology.

\section{DESIGN OF THE TE FILTER}

A waveguide based on a line defect in a pillar PhC can serve as a TE filter if the TE polarized light does not couple into the $\mathrm{PhC}$ waveguide (either if this waveguide has a TE bandgap or if the coupling efficiency for TE is very small) or if the TE polarized light is not confined by the crystal, in which case it radiates away from the line defect. In this letter, we aim for the design and realization of a $\mathrm{PhC}$ waveguide with a high transmission for TM polarization, and a very small coupling efficiency for TE polarization due to a large impedance mismatch. Therefore, the line defect was first optimized for TM transmission, as the transmission of TM polarized light should be high.

Based on 2-D band solver calculations, and using the effective index method to account for the third dimension, the background $\mathrm{PhC}$ was chosen to have a lattice constant $a=491 \mathrm{~nm}$ and a radius $r=0.25 a$. The introduction of a line defect of larger pillars along the $\Gamma X$-direction creates two guided TM modes inside the bandgap of the PhC. Fig. 1 shows the projected band diagram of a $\mathrm{PhC}$ waveguide based on a line defect with radius $r_{d}=210 \mathrm{~nm}$. The mode that increases in frequency, having a positive slope, is a mode with odd symmetry. The mode with a negative slope is a mode with even symmetry. At the wavelength of operation, i.e., in the range from 1530 to $1570 \mathrm{~nm}$, the waveguide only supports the even symmetry mode.

The light is coupled from a conventional ridge waveguide to the $\mathrm{PhC}$ waveguide and vice versa by placing the waveguides next to each other as is schematically shown in Fig. 2. The ridge 


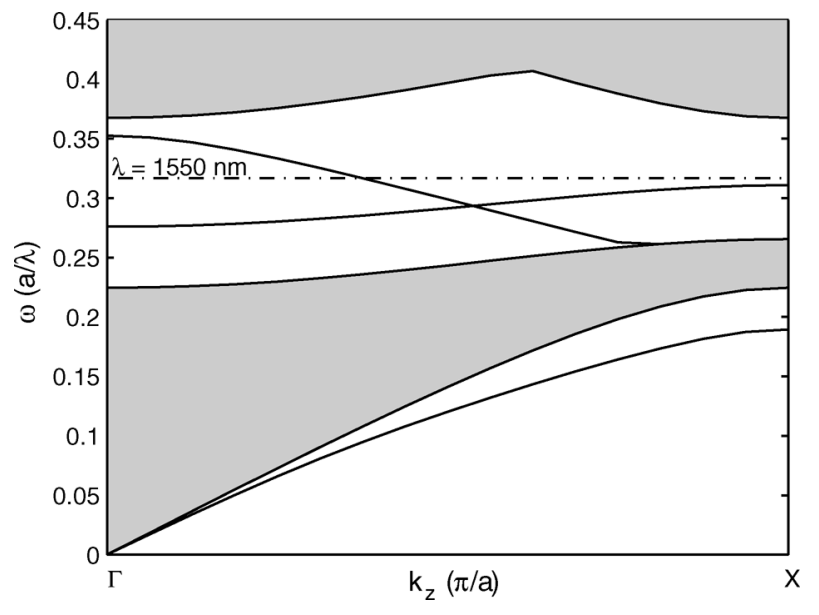

Fig. 1. Projected band diagram of a PhC waveguide consisting of a line of larger pillars in a square lattice showing the TM modes of the waveguide, where the lattice constant is $491 \mathrm{~nm}$, the radius of the rods is $123 \mathrm{~nm}$, and that of the defect pillars is $210 \mathrm{~nm}$.

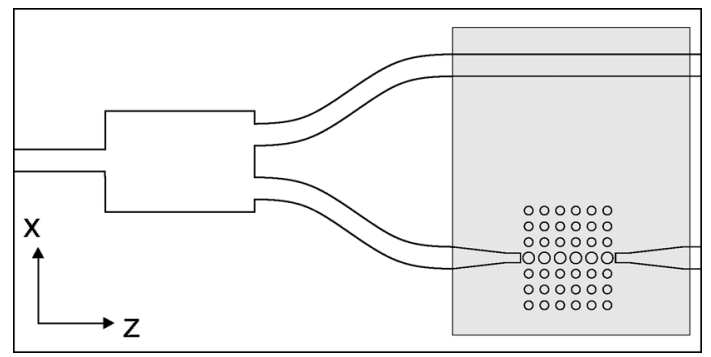

Fig. 2. Schematic drawing of the chip layout. The white area is defined by optical lithography and the gray area by electron beam lithography.

waveguide is first adiabatically tapered down to a width that is equal to the diameter of the defect pillars, i.e., $420 \mathrm{~nm}$. According to simulations, the transmission for TM polarized light is highest if the gap between the end facet of the ridge waveguide and the first PhC pillar is taken to be $36 \mathrm{~nm}$.

In Fig. 3, the calculated transmission is shown for a $\mathrm{PhC}$ waveguide that is eight periods long (based on a three-dimensional (3-D) finite-difference time-domain calculation). The transmission for TM polarized light is $-2.3 \mathrm{~dB}$ with an extinction ratio better than $25 \mathrm{~dB}$ around $\lambda=1550 \mathrm{~nm}$. The good performance of this device is mainly due to a high coupling efficiency of the TM polarization at the transitions between the ridge waveguides and the $\mathrm{PhC}$ waveguide, while the TE polarization has a poor coupling from the access ridge waveguide to the $\mathrm{PhC}$ waveguide. The length of the polarization filter is only $3.9 \mu \mathrm{m}$ (eight times the lattice constant).

The optical circuit design, as schematically shown in Fig. 2, on the chip consisting of a $2-\mu \mathrm{m}$-wide input ridge waveguide, followed by a $1 \times 2$ multimode interference (MMI) coupler splitting the light into two branches. Because of the symmetry of this device, both output arms receive equal optical powers, even if the dimensions deviate from the design parameters. In the reference branch, the light propagates through a conventional ridge waveguide towards the output side of the chip. The other branch contains a PhC waveguide. This configuration has two advantages. First, the coupling alignment into the input waveguide can

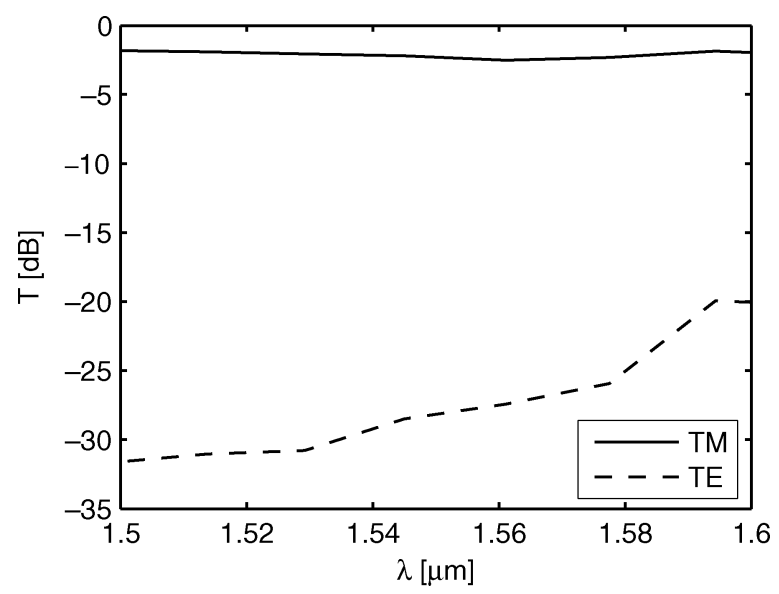

Fig. 3. Calculated transmission of an eight-period-long $\mathrm{PhC}$ waveguide for both TM and TE polarization. The simulation is based on a 3-D FDTD calculation.

easily be optimized using the reference arm. Second, the transmission of the $\mathrm{PhC}$ waveguide can directly be calculated from a comparison with the transmission of the reference arm.

\section{FABRICATION}

The ridge waveguides and the MMIs are defined by optical lithography, whereas the PhCs are defined by electron beam lithography to have sufficient control over the critical dimensions. At the transition between the optically defined waveguides and the e-beam lithography areas, the waveguides are $0.8 \mu \mathrm{m}$ wide. The e-beam lithography section includes the $0.8-\mu \mathrm{m}$ waveguide of the upper arm to account the transition effect between the optically defined section and the e-beam defined section. The alignment accuracy of the e-beam lithography is better than $50 \mathrm{~nm}$. The waveguide pattern, including the PhCs, is first defined in a 50-nm-thick chromium masking layer by a series of lithography steps. This pattern is transferred into a 430-nm-thick silicon dioxide layer by reactive ion etching (RIE) using a $\mathrm{CHF}_{3}$ chemistry. Finally, the deep etch to create the waveguides is performed by inductively coupled plasma (ICP) RIE using a $\mathrm{Cl}_{2}$ : Ar : $\mathrm{H}_{2}$ chemistry [9]. Fig. 4 shows a scanning electron microscope (SEM) image of the PhC structure after the ICP etch. The pillars are $\sim 3.0 \mu \mathrm{m}$ deep. According to simulations, this should be enough to prevent the light from coupling to the substrate modes. Lateral dimensions of the $\mathrm{PhC}$ are well controlled with this fabrication process [10].

\section{TRANSMISSION MEASUREMENTS}

Light from a tunable laser source is coupled into the input waveguide using a microscope objective. The polarization is fixed to TM or TE by use of a polarizer. At the output side, the transmitted light is collected with a lensed fiber. The collected light is measured by a photoreceiver. After optimization of the in- and outcoupling alignment at $\lambda=1550 \mathrm{~nm}$, the tunable laser scans the wavelength from 1530 to $1570 \mathrm{~nm}$ in steps of $0.1 \mathrm{~nm}$.

The cleaved facets of the chip introduce Fabry-Pérot fringes on the measured spectrum. These are averaged out by taking a running average over ten data points of the spectrum. From the 


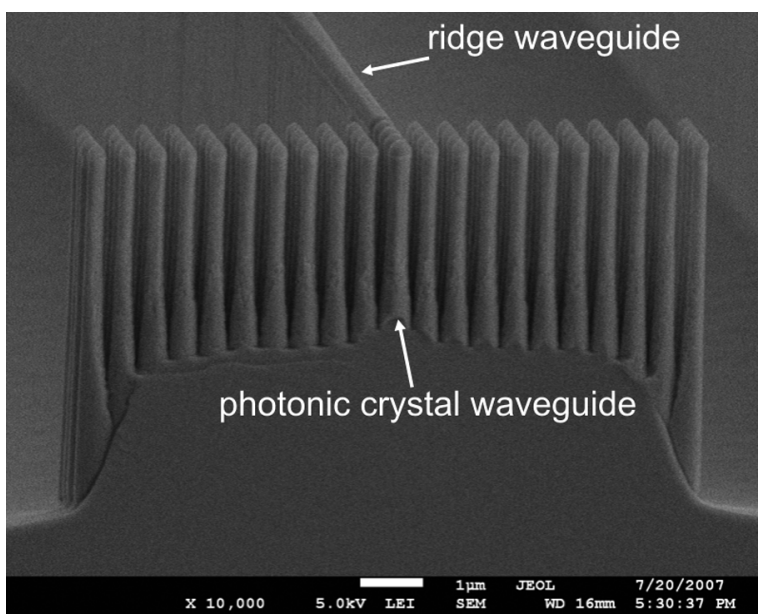

Fig. 4. SEM image of a cross section of a PhC waveguide connected to a classical ridge waveguide on an $\mathrm{InP}$ substrate. In the characterized devices, the silicon dioxide mask layer was removed before the transmission measurements were carried out.

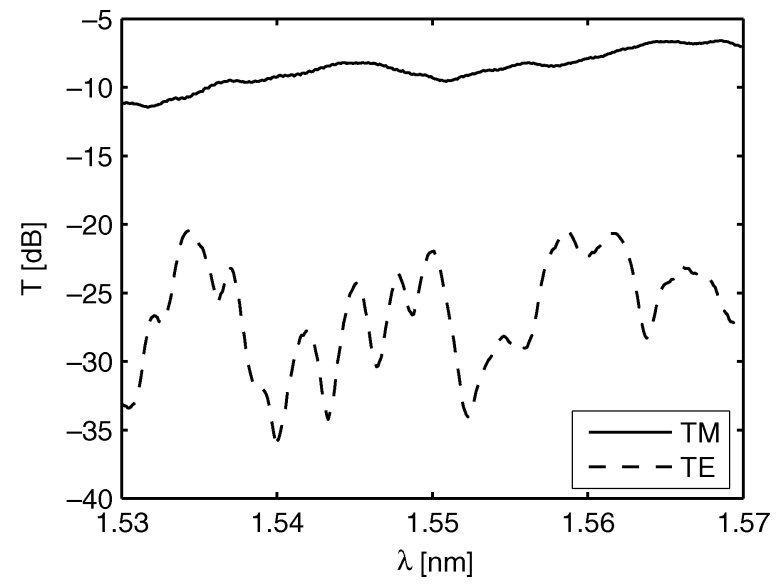

Fig. 5. Measured transmission of a fabricated TE filter for both polarizations. The length of the $\mathrm{PhC}$ waveguide is eight periods $(3.9 \mu \mathrm{m})$.

averaged spectra of both the branches, the transmission of the $\mathrm{PhC}$ waveguide is determined. The transmission losses include the tapering of a waveguide from $0.8-\mu \mathrm{m}$ width down to the diameter of the defect pillars, the coupling to and from the $\mathrm{PhC}$ waveguide, and the propagation loss of the $\mathrm{PhC}$ waveguide.

The measured transmission for both TM and TE polarization is shown in Fig. 5. The average transmission for TM polarized light is $-8.7 \mathrm{~dB}$. The losses are higher than was calculated in the 3-D simulation. Previous results show that coupling losses of the TM polarization are negligible with respect to the propagation losses of the $\mathrm{PhC}$ waveguides [11]. The high propagation loss is probably due to scattering and to the nonvertical sidewalls of the pillars, which can cause large substrate leakage. Both can be reduced by an optimization of the fabrication technology. The TE transmission is $-26.5 \mathrm{~dB}$, which is in agreement with the sim- ulated transmission. The modulation on the transmission of the TE polarization corresponds to a Fabry-Pérot cavity between the transition at the edge of the e-beam write field (where the e-beam lithography is aligned to the optical lithography) and the $\mathrm{PhC}$ waveguide. The reflection at the input of the $\mathrm{PhC}$ waveguide is due to the high impedance mismatch for TE polarization. The reflections at the edges of the e-beam write field can be eliminated by adapting the design of the chip. This implies that an extinction ratio of about $18 \mathrm{~dB}$ should be feasible if the reflections are reduced.

\section{CONCLUSION}

A very short TE polarization filter can be realized in a pillar $\mathrm{PhC}$. The fabrication process is compatible with that of a photonic integrated circuit based on conventional waveguide technology. The device with a length of $3.9 \mu \mathrm{m}$ has a transmission of $-8.7 \mathrm{~dB}$ for TM polarization and $-26.5 \mathrm{~dB}$ for TE polarization. The high losses are probably due to fabrication issues which can be solved by further optimization of the technology. The device, based on a simple design concept, can be used to define the state of polarization in a photonic integrated circuit, improving its stability and performance.

\section{REFERENCES}

[1] T. P. Pearsall, GaInAsP Alloy Semiconductors. Hoboken, NJ: Wiley, 1982.

[2] T. Barwicz, M. Watts, M. Popović, P. Rakich, L. Socci, F. Kärtner, E. Ippen, and H. Smith, "Polarization-transparent microphotonic devices in the strong confinement limit," Nature Photon., vol. 1, no. 1, pp. 57-60, Jan. 2007

[3] L. Augustin, R. Hanfoug, J. van der Tol, and W. de Laat, "A compact integrated polarization splitter/converter in InGaAsP-InP," IEEE Photon. Technol. Lett., vol. 19, no. 17, pp. 1286-1288, Sep. 1, 2007.

[4] J. van der Tol, J. Pedersen, E. Metaal, J.-W. van Gaalen, Y. Oei, and F. Groen, "A short polarization splitter without metal overlays on InGaAsP-InP," IEEE Photon. Technol. Lett., vol. 9, no. 2, pp. 209-211, Feb. 1997.

[5] V. Zabelin, L. Dunbar, N. L. Thomas, R. Houdré, M. Kotlyar, L. O'Faolain, and T. Krauss, "Self-collimating photonic crystal polarization beam splitter," Opt. Lett., vol. 32, no. 5, pp. 530-532, Mar. 2007.

[6] L. Wu, M. Mazilu, J.-F. Gallet, T. Krauss, A. Jugessur, and R. D. L. Rue, "Planar photonic crystal polarization splitter," Opt. Lett., vol. 29, no. 14, pp. 1620-1622, Jul. 2004.

[7] S. Kim, G. Nordin, J. Cai, and J. Jiang, "Ultracompact high-efficiency polarizing beam splitter with a hybrid photonic crystal and conventional waveguide structure," Opt. Lett., vol. 28, no. 23, pp. 2384-2386, Dec. 2003.

[8] J. D. Joannopoulos, R. D. Meade, and J. N. Winn, Photonic Crystals: Molding the Flow of Light. Princeton, NJ: Princeton Univ. Press, 1995.

[9] B. Docter, E. Geluk, M. Sander-Jochem, F. Karouta, and M. Smit, "Deep etched DBR gratings in InP for photonic integrated circuits," in Proc. IPRM, Matsue, Japan, 2007, pp. 226-228.

[10] A. Kok, E. Geluk, B. Docter, J. van der Tol, R. Nötzel, M. Smit, and R. Baets, "Transmission of pillar-based photonic crystal waveguides in InP technology," Appl. Phys. Lett., vol. 91, p. 201109, Nov. 2007.

[11] A. Kok, R. Meneghelli, J. van der Tol, and M. Smit, "Fabrication and characterization of pillar-based photonic crystal waveguides," in Proc. IEEE/LEOS Symp. (Benelux Chapter), Brussels, Belgium, Dec. 2007, pp. $51-54$. 